\title{
Probing ultrafast laser plasma processes inside solids with resonant small-angle $x$-ray scattering
}

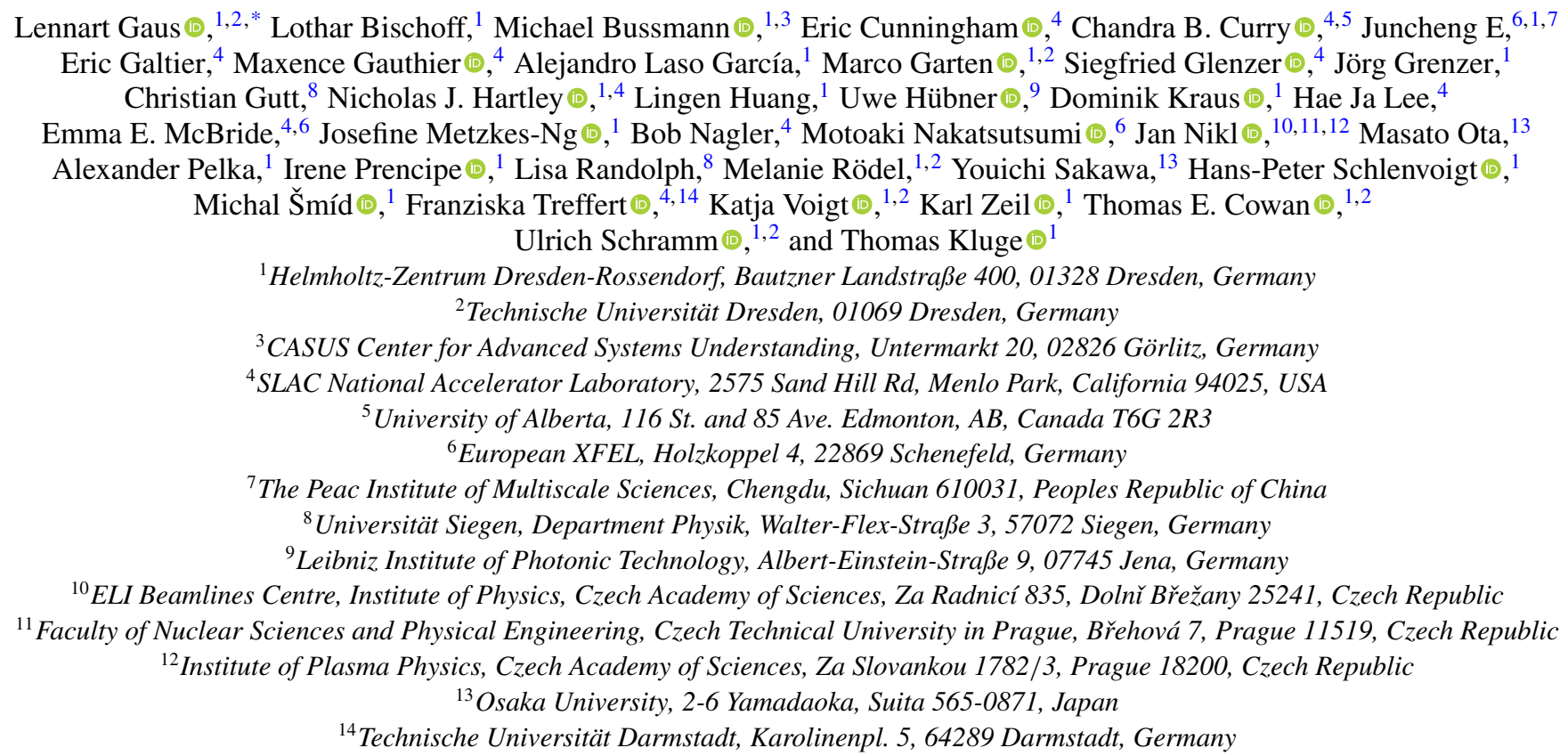

(Received 25 March 2021; accepted 17 November 2021; published 20 December 2021)

\begin{abstract}
Extreme states of matter exist throughout the universe, e.g., inside planetary cores, stars, or astrophysical jets. Such conditions can be generated in the laboratory in the interaction of powerful lasers with solids. Yet, the measurement of the subsequent plasma dynamics with regard to density, temperature, and ionization is a major experimental challenge. However, ultrashort x-ray pulses provided by $\mathrm{x}$-ray free electron lasers (XFELs) allow for dedicated studies, which are highly relevant to study laboratory astrophysics, laser-fusion research, or laserplasma-based particle acceleration. Here we report on experiments that employ a novel ultrafast method, which allows us to simultaneously access temperature, ionization state, and nanometer scale expansion dynamics in high-intensity, laser-driven, solid-density plasmas with a single $\mathrm{x}$-ray detector. Using this method, we gain access to the expansion dynamics of a buried layer in compound samples, and we measure opacity changes arising from bound-bound resonance transitions in highly ionized copper. The presence of highly ionized copper leads to a temperature estimate of at least 2 million Kelvin already after the first $100 \mathrm{fs}$ following the high-intensity laser irradiation. More specifically, we make use of asymmetries in small-angle x-ray scattering (SAXS) patterns, which arise from different spatial distributions of absorption and scattering cross sections in nanostructured grating samples when we tune an XFEL to atomic resonant energies of copper. Thereby, changes in asymmetry can be connected with the evolution of the plasma expansion and ionization dynamics. The potential of XFELbased resonant SAXS to obtain three-dimensional ultrafast, nanoscopic information on density and opacity may offer a unique path for the characterization of dynamic processes in high energy density plasmas.
\end{abstract}

DOI: 10.1103/PhysRevResearch.3.043194

\section{INTRODUCTION}

High energy (HE) and high intensity (HI) lasers can compress and heat solids to extreme states of warm dense matter

\footnotetext{
*1.gaus@hzdr.de

Published by the American Physical Society under the terms of the Creative Commons Attribution 4.0 International license. Further distribution of this work must maintain attribution to the author(s) and the published article's title, journal citation, and DOI.
}

(WDM) and high energy density plasmas (HEDP), important for planetary science [1] and astrophysics [2], fusion energy research [3], the investigation of material properties [4], as well as radiative properties of plasmas [5] and atoms, e.g., in NEET/NEEC processes [6,7], but also important for fundamental research of relativistic plasma physics, HI laser absorption, electron transport, filamentation, and ion channel formation in laser ion acceleration experiments [8].

Understanding and controlling fundamental interaction processes of lasers and solids such as absorption at the solid-density surfaces, ultrafast growth of instabilities, or twostream unstable electron transport as well as compression and 
heating in both HE [9] and HI laser solid interaction [10-12] require measurements with high spatial and temporal precision.

In order to measure the above mentioned processes and develop HI laser-based applications, as well as to open up new possibilities for fundamental relativistic plasma physics by enabling direct comparison to models and simulations, it is necessary to probe the plasma on the fundamental scales of down to a few nanometers and femtoseconds. The only source that has been shown to achieve this in solid-density plasmas currently are X-ray free-electron lasers (XFELs) [13-15]. The ultrashort pulses of hard $\mathrm{x}$ rays produced by XFELs provide the necessary penetration power, and high spatial and temporal resolution for pump-probe experiments of laser-solid interaction processes. The development of novel probing capabilities to fully exploit the large-scale XFEL experiments is an important and fundamental topic. Small-angle $\mathrm{x}$-ray scattering (SAXS) is a unique method that offers a resolution down to the few nanometer scale that is ideally suited for solid-density probing[16-18]. Thereby, it fills the gap between the resolutions of subnanometers for wide-angle $\mathrm{x}$ ray scattering and hundreds of nanometers for phase-contrast imaging [19], where also optical techniques [20-22] that provide few fs and few $\mathrm{nm}$ resolution in low density plasmas fail due to the impermeability of solids.

With SAXS, the complex plasma dynamics becomes accessible via the temporal evolution of the nanoscale Fourier components of the scattering length density $\rho(\boldsymbol{r}, t)=n_{e}+$ $n_{i} f_{i}$, where $n_{e}$ and $n_{i}$ are the free electron and ion densities, respectively, and $f_{i}$ is the ion form factor. The use of structured targets has so far proven to be particularly useful and sensitive to nanoscopic, ultrafast dynamics of the plasma electron density $\left(n_{e}\right)$ employing SAXS [16,17]. In the present work we also measure in small-angle transmission geometry, but here we take advantage of a different, so far ignored, property of the scattering patterns, namely the asymmetry. This enables us to measure the ionic contribution to the scattering, which is primarily given by the imaginary part of $\rho$ via x-ray absorption, e.g., in bound-bound transitions. This means we gain access to the plasma opacity simultaneously to the plasma density distribution via (resonant) SAXS.

Tuning the narrow-bandwidth XFEL photon energy to a specific atomic bound-bound transition energy allows us to turn on and off the asymmetry effect [8]. Since the opacity depends on the existence of specific ionization states in the plasma, which in turn depend on the plasma temperature, with according modeling this method resembles an ultrafast in situ temperature measurement, similar for example to x-ray Thomson scattering (XRTS) [23] but with the potential of high spatial resolution similar to the element-specific imaging technique presented by C. Song et al. [24].

We demonstrate that the asymmetry arising from differences in the distributions of electrons and ions can be used to reconstruct spatial correlations between three-dimensional (3D) structures and their respective material properties.

\section{ASYMMETRY IN THE SCATTERING PATTERNS}

SAXS patterns, being proportional to the absolute square of the Fourier transform of the scattering length density $\rho$, are usually symmetric since $\rho$ is often either purely real valued, purely imaginary, or the real and imaginary parts are proportional to each other. Hence, any asymmetry

$$
\eta(\boldsymbol{q})=\frac{I(\boldsymbol{q})-I(-\boldsymbol{q})}{I(\boldsymbol{q})+I(-\boldsymbol{q})}
$$

in a SAXS intensity pattern $I(\boldsymbol{q})$, where $\boldsymbol{q}$ is a vector in the reciprocal space, can be directly linked to the different distribution of the real and imaginary parts of $\rho$ in the target (see the Supplemental Material, Sec. V [25]). In the case of hard $\mathrm{x}$ rays, the real part is primarily due to Thomson scattering, while the imaginary part arises from the opacity, i.e., the combined absorption cross section of atomic resonant boundbound (bb), bound-free (bf), and free-free (ff) transitions. In the scattering length density the opacity enters via complex valued optical corrections $f_{i}^{\prime}+i f_{i}^{\prime \prime}$ that need to be added to the ion form factor $f_{i}$.

The samples used in this experiment are one-dimensional (1D) (multilayer) grating targets with structures positioned at different depths (see Fig. 1), which yield a high signal at high $q$ components that correspond to $\mathrm{nm}$ resolution. The prestructured targets provide a comparably clean initial condition and allow us to follow the changes induced by the rapid plasma dynamics via the pump-probe technique, specifically here the buried layer heating and expansion $[8,26]$, and laser absorption at structured surfaces [27].

In transmission probe geometry, the Fourier components from structures at different depths superimpose on the detector so that single-pulse SAXS experiments so far give access to the two-dimensional (2D) areal density distribution only.

Both the grating and buried grating targets we employed in our experiments have two grated interfaces. We can express the two structured contours of the front (or buried) and rear surface interface as $z_{1}(\boldsymbol{r}, t)$ and $z_{2}(\boldsymbol{r}, t)$, respectively. Correlations between both contours are expressed in reciprocal space by a replication factor $\chi(\boldsymbol{q}, t)$ with

$$
\chi(\boldsymbol{q}, t)=\frac{\tilde{z}_{2}(\boldsymbol{q}, t)}{\tilde{z}_{1}(\boldsymbol{q}, t)} .
$$

A replication factor of $|\chi(\boldsymbol{q}, t)|=1$ means perfect replication, i.e., the two interfaces have identical profiles.

For complex form factors, it follows from the Fourier transform symmetry properties that the scattering intensity is asymmetric for two or more materials being asymmetrically distributed with respect to each other. In the Supplemental Material, Sec. V [25], we show an intuitive example and demonstrate how to calculate the asymmetries for the target configurations relevant for our experiments. The main finding is that for two-layer targets the replication factor $\chi(\boldsymbol{q})$ and the respective optical properties of the target materials can be determined from the asymmetry in the scattering patterns via

$$
\eta(\boldsymbol{q}, t)=\frac{c_{1} \operatorname{Im}[\chi(\boldsymbol{q}, t)]}{\operatorname{Re}\left[c_{2}+c_{3} \chi(\boldsymbol{q}, t)^{2}+c_{4} \chi(\boldsymbol{q}, t)\right]}
$$

if the phase of the XFEL is plane [28]. This equation connects the asymmetry with the structural parameters (entering in $\chi)$ and the material properties such as density and opacity (entering in $c_{i}$ defined in the Supplemental Material [25]). 


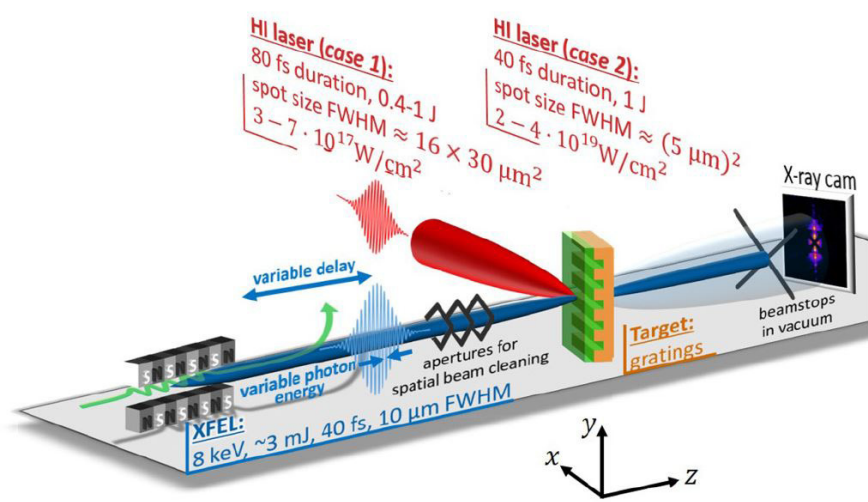

(a)
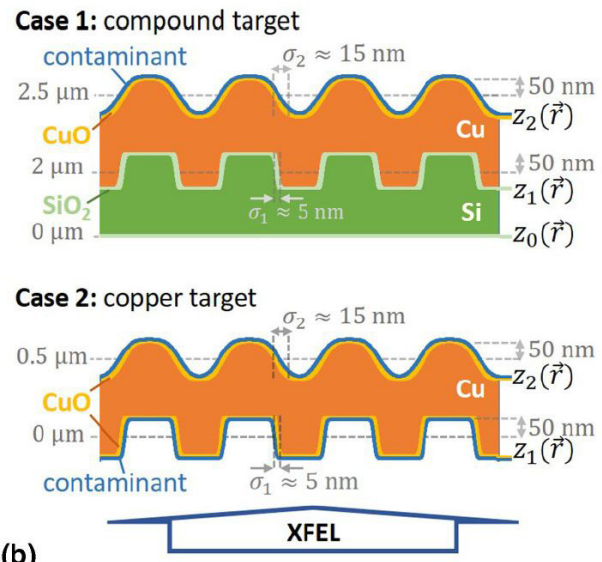

(b)

FIG. 1. Schematic of the resonant-SAXS experimental setup (a) and target geometry (b) (not to scale). A near infrared HI laser pulse is focused onto a silicon-copper compound target with a grating interface (case 1) or copper grating (case 2). The profile edges are assumed to follow an error function $z \propto \operatorname{erf}\left[\left(y-y_{0}\right) /\left(\sqrt{2} \sigma_{1 / 2}\right)\right]$. Both the HI drive laser and XFEL probe beam axes are oriented at $45^{\circ}$ with respect to the target surface normal, parallel to the grating ridges and polarized as shown.

A simple way to break the symmetry is a shift of the rear layer by $\boldsymbol{r}_{s}$ with respect to the front layer structure, e.g., by a tilt of the target: $\chi(\boldsymbol{q})=e^{-i \boldsymbol{r}_{s} \boldsymbol{q}}$. In experimental conditions, surface inhomogeneities (i.e., random variations in the geometric parameters) and contamination layers of oxides and hydrocarbon compounds are unavoidable and can generate asymmetry. Their influence can be absorbed into the parameters $c_{i}$, as demonstrated in the Supplemental Material [25]. In fact, for the targets used in this paper, the random variations do not cancel out due to their number not being large enough, hence breaking the symmetry. They are the leading cause of the asymmetry and are taken into account in the calculations for the expected asymmetries of our targets which are used later in Figs. 5 and 7(f).

Before we present our results regarding the opacity measurements, we need to study the role of the replication factor. In fact, we will demonstrate that the asymmetry can be used to obtain nanoscopic information about interface structures lying behind each other. We performed dedicated experiments for both cases: Case 1 is an experiment measuring the relative expansion dynamics of interface structures placed at different depths. Here the laser pump intensity was chosen small enough that only structural changes occur, i.e., only the replication factor $\chi$ varies while the optical properties of the compound materials remain constant. In case 2 we focus on changes in the asymmetry caused by variations of the optical properties of the target material (via $c_{i}$ ). Here a combination of high pump laser intensity and short timescales yield a rapid heating, ionization, and consequently an increase of x-ray opacity at resonant bb transitions.

Experimentally we extract the asymmetry by comparing the scattering signal in the scattering peaks for each peak pair at $+q$ and $-q$. The absolutes of the individual asymmetry values are then averaged in order to have a simple quantity to easily compare simulations with theory. Additionally, the uncertainty margins of the averages are much smaller than those of the individual asymmetry values. Figure 2 illustrates the workflow from the raw experimental data to the average asymmetry values $\overline{|\eta|}$.

\section{EXPERIMENTAL RESULTS}

\section{A. Case 1: Geometric changes}

For this part of the experiment, $\mathrm{Si}-\mathrm{Cu}$ compound targets were pumped by the HI laser at an intensity of 3 and $7 \times$ $10^{17} \mathrm{~W} / \mathrm{cm}^{2}$, respectively (see Fig. 1). At this comparatively low intensity, the resulting heating is sufficiently low such that collisional ionization of the rear copper layer is suppressed to less than the $L$ shell, i.e., no $K \alpha$ transition channels are available. Additionally, a slow effective expansion of either one or both the buried grating interface and rear grated surface (i.e., an increase of $\sigma_{1}$ or $\sigma_{2}$ ) can be deduced from a decrease of the scattering yield at large values of $q$ with a

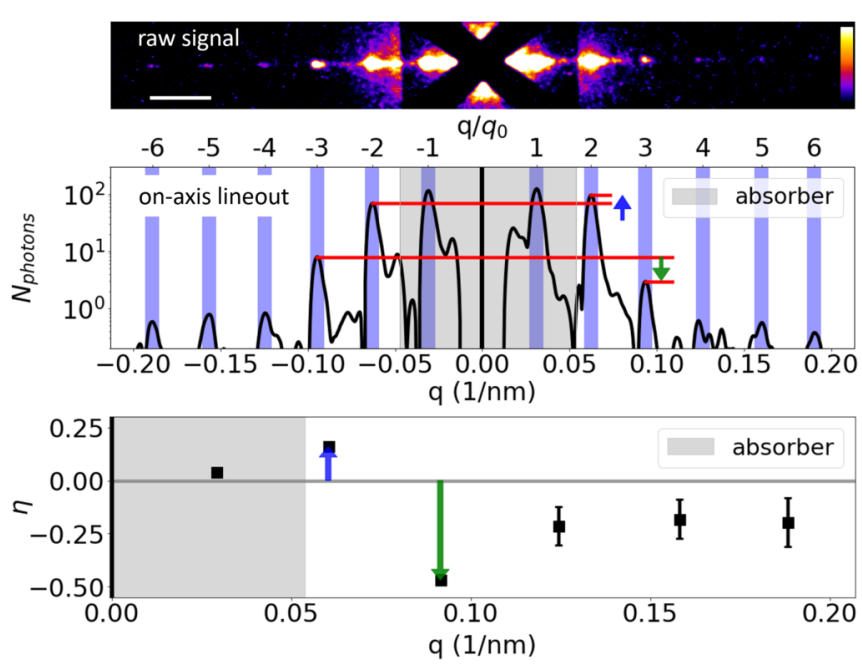

FIG. 2. Data processing steps from raw signal to asymmetry on the example of a resonantly probed $\mathrm{HI}$ laser driven $\mathrm{Cu}$ grating foil. Top: Raw data (log color scale), white bar corresponds to $0.03 \mathrm{~nm}^{-1}$; middle: background corrected lineout through center, blue bars indicate the $q$ ranges for integration of the photon number per peak; bottom: asymmetry of peak pairs whose averaged absolute values

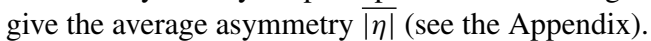




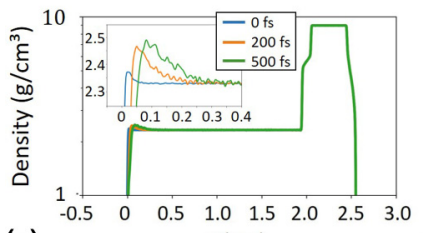

(a)

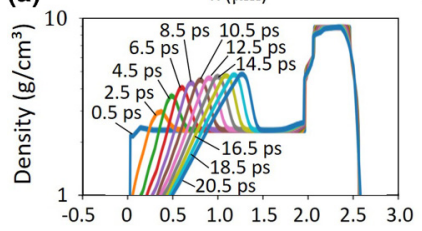

(c)

$x(\mu \mathrm{m})$

(d)

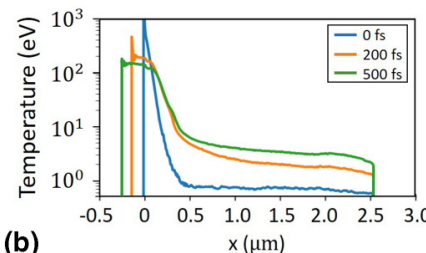

(b)

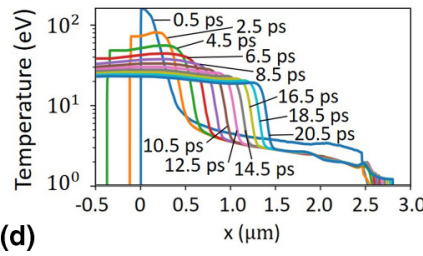

FIG. 3. (a) and (c) Density and (b) and (d) temperature evolution of the $\mathrm{Si}-\mathrm{Cu}$ compound target for the average optical laser intensity in the XFEL probe area from particle in (a) and (b) cell simulations and (c) and (d) hydrodynamic simulations. Laser strength was set to $a_{0} \cong 0.3$, which corresponds to the average intensity in the XFEL focal spot for $1 \mathrm{~J}$ laser energy. Laser is incident from the left.

Debye-Waller-like procedure similar to that presented in [17]. Comparing the SAXS patterns obtained after irradiation by the HI laser pulse (main shots) with those obtained before from the target without HI irradiation (preshots), we observe a reduction of scattering yield at large $q$ values corresponding very roughly by an effective expansion of up to $10 \mathrm{~nm}$ (see the Supplemental Material [25]). However, the expansion values cannot be fitted separately for the interface and rear surface with a reasonable accuracy since the projected total electron density of the target with two grating surfaces simply has too many free parameters.

On the other hand, no significant reduction of the yield was found for the shortest probe delay of $250 \mathrm{fs}$, indicating that the interface expansion indeed is a slow process over a few ps, as expected for those low laser intensities.

To confirm the expectations of low heating and slow expansion, we performed particle-in-cell (PIC) simulations to model the laser interaction and prompt electron heating in the first 500 fs after the laser hit the target, followed by a hydrodynamic simulation to study the plasma response over the few ps range up to the 16 ps delay of the latest probe, see Fig. 3. While at the front surface the high electron temperature generates a strong ablation pressure which leads to shock formation and ablation, heat diffusion towards the target rear leads to a partial temperature equilibration over the whole compound target depth to $5 \mathrm{eV}$ within the following few hundred femtoseconds. The key finding here is that the PIC simulation predicts a very slow expansion of the buried interface and the rear surface, which is in fact below the simulation's resolution within the first 500 fs. With the hydrodynamic simulations we then observe the formation of a strong shock over the next 5 ps that propagates toward the target rear with an initial velocity of $169 \mathrm{~nm} / \mathrm{ps}$, slowing down to $45.5 \mathrm{~nm} / \mathrm{ps}$ after $20 \mathrm{ps}$. This means that the rear side interface remains intact during the whole range of our probing delays and scattering patterns are changing only due to the thermal expansion of the grating surfaces. We now analyze the asymmetry parameter $\overline{|\eta|}$ in the experimental scattering patterns in order to obtain additional information about the

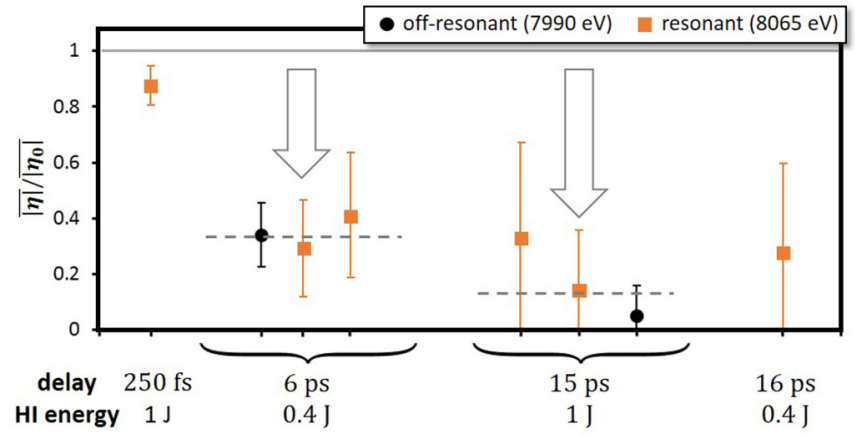

FIG. 4. (Case 1) Average asymmetry $\overline{|\eta|}$ relative to that of the preshots $\overline{\left|\eta_{0}\right|}$ for single shots on $\mathrm{Si}-\mathrm{Cu}$ compound targets with 3-7 $\times$ $10^{17} \mathrm{~W} / \mathrm{cm}^{2}$ laser intensity. Dashed lines are respective weighted averages.

relative expansion of both gratings. We observe a reduction of $\overline{|\eta|}$ compared to its value $\overline{\left|\eta_{0}\right|}$ in the respective XFEL-only preshots, which is dependent on a combination of the delay and energy of the HI laser (see Fig. 4). As expected, since no $K \alpha$ channels are available, the reduction of the asymmetry is not substantially different for the different XFEL photon energies (i.e., on- and off-resonance). The observed reduction of the asymmetry must therefore be predominantly due to a change of the replication factor, i.e., expansion of the grating surfaces.

In Fig. 5 the calculated asymmetries (see the Supplemental Material, Sec. V [25]) are shown as a function of the two respective smoothness parameters $\sigma_{1}$ and $\sigma_{2}$ of the buried and rear grating ridges. We adopt a replication factor of

$$
\chi(\boldsymbol{q})=\hat{\chi} \exp \left(-\Delta \sigma^{2} q^{2} / 2\right)
$$

with $\Delta \sigma^{2} \equiv \sigma_{2}^{2}-\sigma_{1}^{2}$, i.e., a smoothing of the rear interface (index 2) with respect to the buried interface (index 1). With the initial conditions for the unpumped targets (see the Appendix), it can be readily seen that a reduction in asymmetry is linked to an expansion of the grating interfaces. Specifically, the experimentally observed reduction by more than a factor of 3 reduces the range of possible values for $\sigma_{1}$ and $\sigma_{2}$. The

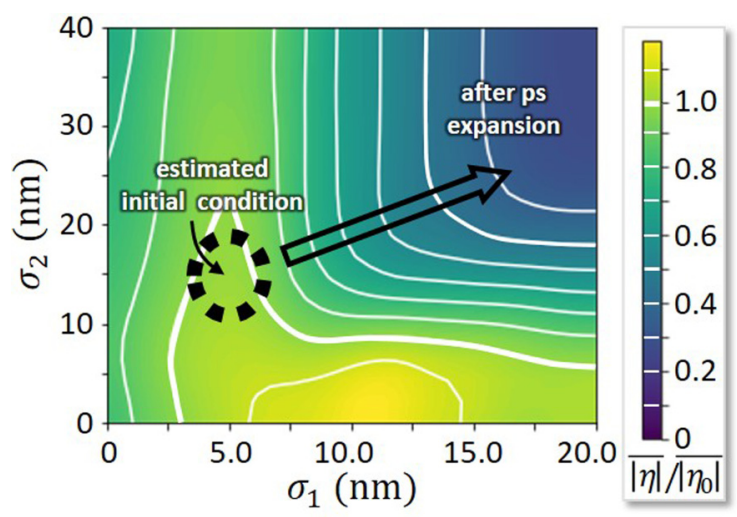

FIG. 5. Analytical modeling of the asymmetry $|\bar{\eta}|$ averaged over the experimentally accessible $q$ range and normalized by the corresponding preshot asymmetry $\overline{\left|\eta_{0}\right|}$ for case 1 ( $\mathrm{Si}-\mathrm{Cu}$ compound targets). 


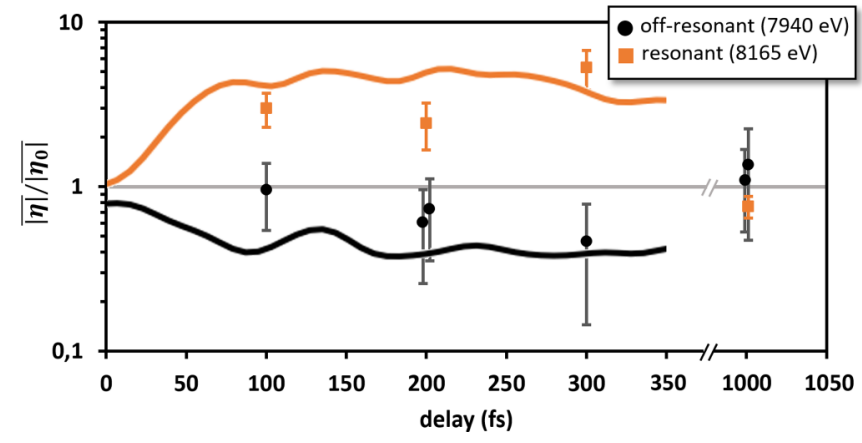

FIG. 6. (Case 2) Average asymmetries $\overline{|\eta|}$ relative to that of the preshots $\overline{\left|\eta_{0}\right|}$ for the shots on $\mathrm{Cu}$ targets with $2-4 \times 10^{19} \mathrm{~W} / \mathrm{cm}^{2}$ laser intensity. Solid lines show asymmetry obtained from PIC simulations for off-resonant (black) and resonant (orange) XFEL energy. Vertical error bars were obtained by Gaussian error propagation (see the Appendix). The delay time error of $\pm 114 \mathrm{fs}$ is given by the rms jitter of the XFEL.

result we can therefore infer is that the observed drop in asymmetry is characteristic for the expansion of the buried grating interface $\left(\sigma_{1}\right)$ by at least $10 \mathrm{~nm}$ and cannot be explained by an expansion of the rear grating interface $\left(\sigma_{2}\right)$ alone.

\section{B. Case 2: Opacity changes}

The second case considered in this paper focuses on ionization-induced changes in the asymmetry of the SAXS signal as a novel tool to measure the temporal evolution of plasma opacities. For this purpose we used $\mathrm{Cu}$ grating targets irradiated by a higher laser intensity with a peak intensity of $(3 \pm 1) \times 10^{19} \mathrm{~W} / \mathrm{cm}^{2}$. With the first grating positioned now directly at the front surface, the dynamics are no longer dominated by heat diffusion and shock propagation through the silicon layer as before in case 1 , but by the faster direct laser-solid interaction and by hot electrons accelerated into the target by the laser. The relevant timescales for these processes are shorter, on the order of a few hundred femtoseconds only. Consequently, we start to observe a slight decrease of the asymmetry due to the grating expansion for shots with XFEL probe delay already at 200 fs at off-resonant probe energies of 7940 eV, see Fig. 6.

For resonant scattering we tuned the energy of the XFEL to an open atomic transition in the plasma, namely to $8165 \mathrm{eV}$. This is the energy of the $K \alpha$ transition in nitrogenlike $\mathrm{Cu}$ (K1L6-K2L5) [29]. During the first 300 fs after pump laser irradiation, the scattering patterns in those resonant shots exhibited large values of asymmetry compared to the XFELonly preshots, cf. Fig. 6. The increase in asymmetry only disappears at larger delays. At the largest delay of $1 \mathrm{ps}$ the main shots and preshots show a similar asymmetry again. This is to be expected, due to the onset of expansion/destruction of the front side grating and ion-electron recombination (see below). In order to understand these observations, we refer to the discussion in the previous case 1 . We expect the rear side grating to remain virtually unchanged, while the front surface should thermally expand during the first few hundred femtoseconds after the laser irradiation, which is also supported by PIC simulations described later. Consequently, the reduced asymmetry in the main shots at off-resonant XFEL energy during the initial $300 \mathrm{fs}$ after the HI irradiation compared to the preshots can be explained by a reduced replication factor $\chi$. On the other hand, this means that the increase of asymmetry for the on-resonance shots at the same delays cannot be caused by geometric changes and hence must be due to a change of the opacity at the resonant energy. Since the cross section of bf transitions is similar within the range of the two XFEL photon energies used in the experiment (within $\pm 25 \%$ ), the large increase of asymmetry in the on-resonance shots must be due to an increase of $f_{\mathrm{Cu}}^{\prime \prime}$ due to bb transitions [30], whose existence was measured independently with a temporally and spatially integrating spectrometer (see Fig. 9 in the Appendix).

We therefore conclude that the apparent plasma temperature is above $300 \mathrm{eV}$, which is the necessary temperature to sufficiently ionize copper to open the nitrogenlike $K \alpha$ resonances at the probe photon energy of $8165 \mathrm{eV}[29,31]$. However, the plasma evolution is more complex, i.e., nonthermal and highly transient, as the laser-plasma interaction is pulsed, relativistic, and nonlinear. In order to model the plasma evolution, we performed a set of PIC simulations. We varied the relativistic laser amplitude $a_{0}$ to model different regions inside the UHI laser focal spot.

During the first few tens to hundreds of femtoseconds following the HI laser irradiation, the grating is quickly ionized and electrons are accelerated and heated. The simulations for $a_{0}=3.7$ down to $a_{0}=1.2$ show average free electron energies in the range of multiple $\mathrm{keV}$; for the nominal average intensity in the grating area, i.e., $a_{0}=2.1$, it peaks at approximately $\langle E\rangle=5 \mathrm{keV}$, cf. Fig. 7. It is worth mentioning that in thermal equilibrium an electron temperature in this range would suggest that $\mathrm{Cu}$ should be ionized up to $\mathrm{He}$ - and $\mathrm{H}$-like states. Consequently, we could not expect to observe N-like $K \alpha$ transitions. However, due to the nonequilibrium transient situation and limited ionization rates, the average ionization can be seen and is known [32] to be much less during the first several hundred femtoseconds. Thus, the ionization distribution contains significant contribution of $\mathrm{N}$-like ionized $\mathrm{Cu}$ and the respective $K \alpha$ resonance in fact can be driven by the XFEL.

In order to estimate the increase of absorption and asymmetry, we fit the average charge state of a zero-dimensional atomic simulation (using SCFLY [31], varying the NLTE temperature) to that of the PIC simulation for each time step. From this we can then extract the opacity for the respective matched cases and consequently estimate the temporal evolution of $f_{\mathrm{Cu}}^{\prime \prime}$ [see Fig. 7(e)]. Finally, we can now calculate the expected change of the asymmetry in the SAXS scattering patterns when the XFEL energy is tuned to the nitrogenlike $K \alpha$ resonance energy of $8165 \mathrm{eV}$. For specific expansions of the grating surfaces extracted from the PIC simulations, we can use Eq. (3) with the appropriate extension for including surface contaminants to compute the asymmetries for the respective increased absorption, see Fig. 7(f) for a specific example at the average laser intensity. The predictions for the experimental results have to take into account the spatial laser intensity profile. We do this in Fig. 6 by first computing the asymmetric scattering amplitudes for each of the simulations with different laser intensity. We then calculate the total 

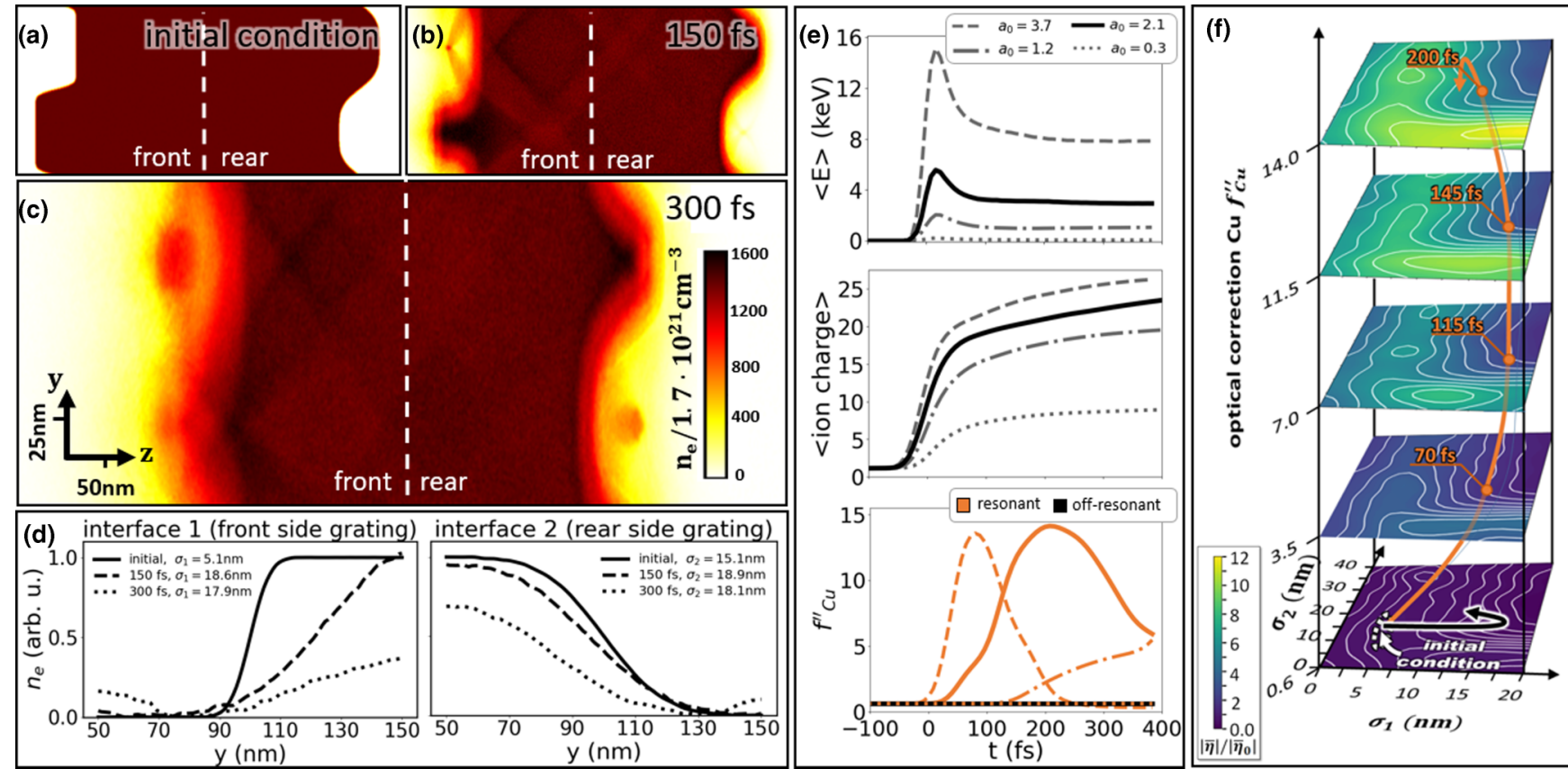

FIG. 7. Simulation of the $\mathrm{Cu}$ target (case 2) for the average intensity of $1.3 \times 10^{19} \mathrm{~W} / \mathrm{cm}^{2}\left(a_{0}=2.1\right)$. (a)-(c) Total electron density distribution at three different times. (d) Transverse electron density integrated through the first (left) and the rear half of the target (right) normalized with respect to the initial grating amplitude. (e) Temporal evolution of the average electron energy (top), average Copper ionization charge state (middle), and fitted opacity using SCFLY [31] (bottom) for resonant (orange, 8165 eV) and off-resonant (black, 7940 eV) XFEL energy. For reference, the evolution is given also for different laser intensities, corresponding to different parts of the laser focal spot. (f) Calculated asymmetry based on the simulation results for resonant (orange) and off-resonance (black) XFEL energy.

scattering signal by summing up those individual contributions, weighted by the areal fraction of the respective laser intensity in the focal spot. The resulting semianalytical predictions for the asymmetries of the scattering pattern can be seen to be in excellent agreement with the experimental results.

Summarizing, the fact that the asymmetry is increased during the first $300 \mathrm{fs}$ after HI laser irradiation is due to a combination of electron acceleration to an average energy well above $300 \mathrm{eV}$, moderate delayed grating expansion, and comparatively slow ionization not reaching saturation during the brief heating time before the bulk cools down again.

\section{DISCUSSION AND OUTLOOK}

To our knowledge, this is the first published experiment that has combined an XFEL with a relativistically intense laser beam in order to generate and probe hot dense plasmas. The potential of XFEL-based SAXS and especially resonant SAXS to measure the spatial distribution of the electron density and plasma opacity as well as correlations both in the XFEL transverse and longitudinal direction via the replication factor offers a unique way to characterize complex dynamic plasma processes. We developed an analytical model that demonstrates the connection between the optical properties of a material and surface replications in the $\mathrm{x}$-ray longitudinal direction with the asymmetry in the scattering pattern. Here we demonstrated the application of asymmetric scattering on disentangling the expansion of a buried layer from the one at the rear, and to extract the plasma opacity by tuning the XFEL energy to the nitrogenlike bb transition of copper. Using an idealized target model including random surface contamination, the asymmetry changes upon laser pumping were found to be in quantitative agreement with an expansion of a grating buried $2 \mu \mathrm{m}$ deep in silicon of at least $10 \mathrm{~nm}$ at low laser intensities, and a heating of a thin copper target to at least $300 \mathrm{eV}$ at $\mathrm{HI}$ laser intensities exceeding $10^{19} \mathrm{~W} / \mathrm{cm}^{2}$.

Here we use simple 1D grated targets in longitudinal transmission geometry. However, this is part of our broader efforts to develop structured targets as a platform for HED XFEL experiments. One could easily envision longitudinal structures and transverse probing, chirped gratings, or complex 2D structures buried for example inside the target. The principles of resonant SAXS can also be transferred to grazing incidence SAXS or other scattering geometries. This flexibility makes periodically structured targets a versatile experiment platform that can be easily adapted to a wide range of science cases to measure the density and opacity gradients on surfaces or inside the compressed and heated solid.

Additionally, grating targets themselves are an interesting research object. Samples with a grated front surface, for example, have shown complete laser absorption resulting in enhanced ion acceleration and extreme ultraviolet (XUV) generation $[27,33]$. Multilayer structures containing few hundred nanometers to micron deep buried structures with nanoscale spatial modulation are ideal for studies of isochoric or shock heating of solid-density plasmas [26], or can serve as an ex situ model of DT layered fusion capsules or to study the role of surface roughness and modulations by adding a grating layer.

Establishing the experiment platform of structured targets is also a stepping stone towards bringing $\mathrm{HI}$ laser relativistic 
plasma fundamental physics experiments to XFELs, e.g., by probing the nanoscopic evolution of structures self-generated during and after the laser irradiation [30,34].

For such complex density distributions or prestructured and stronger signals, in the future it will become possible to make use of the full $\boldsymbol{q}$ dependency of the asymmetry. Then, phase retrieval algorithms can be employed to directly image the complex-valued scattering amplitude [35-37], and to obtain the electron and ionization state spatial distribution separately and model-free in a single shot with a single detector. Additionally, advanced imaging methods such as Fourier transform holography [38] and two-color or x-ray-pulse split-and-delay probing [39] can in the future help to employ the asymmetric scattering for extraction of these parameters.

This technique has applications in a wide range of dynamical phenomena such as laser ablation, laser heating and ionization, shock formation in warm dense matter, plasma expansion, or plasma instabilities. It may enable the exploration of the opacity on the nanometer and femtosecond scale in nonthermal conditions, which can be used as an ultrafast high resolution thermometer for warm and hot dense matter. Moreover, with access to longitudinal correlations in transmission geometry and high temporal resolution, it can assist tomographic methods to gain a 3D understanding of the sample structure in highly dynamical systems.

The raw data used for this publication is available under GNU Lesser General Public License 3.0 [40].

\section{ACKNOWLEDGMENTS}

This work was partially supported by DOE Office of Science, Fusion Energy Science under FWP 100182. Use of the Linac Coherent Light Source (LCLS), SLAC National Accelerator Laboratory, is supported by the U.S. Department of Energy, Office of Science, Office of Basic Energy Sciences under Contract No. DE-AC02-76SF00515. The experiments were performed at the Matter at Extreme Conditions (MEC) instrument of LCLS, supported by the DOE Office of Science, Fusion Energy Science under Contract No. SF00515. This work has also been supported by HIBEF [41] and partially by Horizon 2020 LASERLAB-EUROPE (Contract No. 871124) and by the German Federal Ministry of Education and Research (BMBF) under Contract No. 03Z1O511. This work was partially funded by the Center of Advanced Systems Understanding (CASUS), which is financed by Germany's Federal Ministry of Education and Research (BMBF) and by the Saxon Ministry for Science, Culture and Tourism (SMWKT) with tax funds on the basis of the budget approved by the Saxon State Parliament. Portions of this research were carried out at ELI Beamlines, a European user facility operated by the Institute of Physics of the Academy of Sciences of the Czech Republic. Supported by CAAS project CZ.02.1.01/0.0/0.0/16_019/0000778 from European Regional Development Fund; Czech Technical University Grant No. SGS19/191/OHK4/3T/14 and Czech Science Foundation project 19-24619S. The computations were performed using computational resources funded from the CAAS project. This work has received funding from the Eurofusion Enabling Research Project No. CfP-FSD-AWP21-ENR-01-
CEA-02. C.G. acknowledges funding via DFG GU 535/6-1. This research was partially supported by Japan Society for the Promotion of Science (JSPS) KAKENHI Grants No. JP17H06202.

\section{APPENDIX: METHODS}

\section{Target samples}

For the experiments we used grated layer targets [schematic drawing in Fig. 1(b) of the main text and scanning electron microscopy (SEM) images in Fig. 8 below]. For case 1 we employed compound gratings of a $2 \mu \mathrm{m}$ thin silicon support membrane $\left(n_{e}=7 \times 10^{23} \mathrm{~cm}^{-3}, f^{\prime \prime}=0.33\right)$ covered with a copper layer $\left(n_{e}=2.4 \times 10^{24} \mathrm{~cm}^{-3}, f^{\prime \prime}=0.63\right)$. First, a grating was inscribed into the rear surface of the Si membrane and subsequently covered with copper of a few hundred nanometers thickness. With this the buried grating structure at the $\mathrm{Si}-\mathrm{Cu}$ interface imprints also at the $\mathrm{Cu}$-vacuum surface [see inset in Fig. 1(a) of the main text] yielding a replication factor $\chi(\boldsymbol{q})=\hat{\chi} \exp \left[-\left(\sigma_{2}^{2}-\sigma_{1}^{2}\right) q^{2} / 2\right]$ between the buried and rear side grating structures. Note that the grating interface is protected from direct laser interaction by the Si membrane. For case 2 this membrane was etched away almost completely, leaving only the copper foil with a sharp grating at the front and its less sharp imprint on the rear.

The target grating smoothness $\sigma_{1}$ for the buried grating of the silicon-copper compound targets and the front grating of the pure copper targets were taken from the preshots in [17], as there we used the same grated silicon carrier membrane. There, the grating smoothness parameter was measured to be
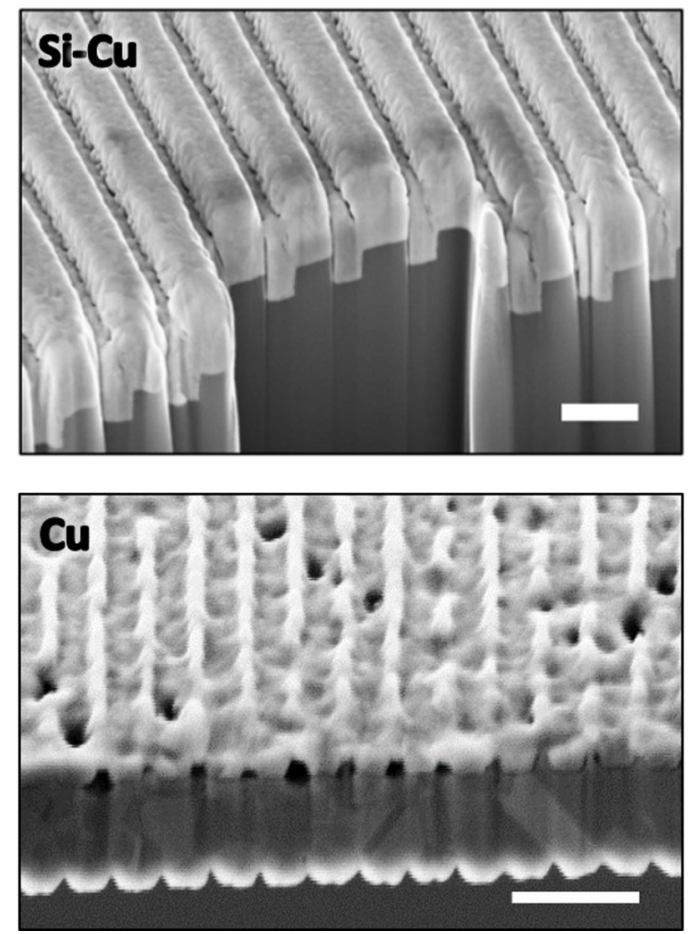

FIG. 8. Scanning electron microscopy (SEM) images of representative target sample cross sections, prepared by focused ion beam cuts. Top: $\mathrm{Si}-\mathrm{Cu}$ - dark: $\mathrm{Si}$, light gray: $\mathrm{Cu}$; bottom: $\mathrm{Cu}$. White bar is $500 \mathrm{~nm}$ 
$5.4 \mathrm{~nm}$. The rear surface smoothness $\sigma_{2}$ of the Cu coating in the present experiment cannot be accessed independently via SAXS, yet we can restrain the possible value of $\sigma_{2}$. It must be larger than $\sigma_{1}$ and based on the analytic analysis of the asymmetry, the results are consistent with a smoothness of $\sigma_{2} \approx 10-15 \mathrm{~nm}$ and $\hat{\chi}=1$, which is also supported by the SEM images (Fig. 8). The buried grating height was set to the manufacturing specification of $100 \mathrm{~nm}$.

The asymmetry was analytically modeled for Figs. 5, 6, and 7(f) based on Eq. (2) and is discussed in the Supplemental Material, Sec. V [25]. As is shown there, the asymmetry is due to the existence of hydrogen and oxides on the target surface and the small randomness in their thickness. However, the asymmetry is not very sensitive to their degree of expression. Their existence can be experimentally shown by the data recorded separately by a Thomson parabola spectrometer, see the Supplemental Material [25]. The exact thickness cannot be determined by this method but their approximate values can be extracted from literature [42-44]. In principle, defects or other geometric effects on the nanometer level that we resolve with SAXS are deviations from our simple grating model and can potentially introduce or change the asymmetry. However, any defects that change the asymmetries of our measurements need to have a correlation on the scale of the grating period, or else their effect on the scattering signal at the peak positions will be greatly reduced as compared to the effect of the surface contaminations which are correlated greatly with the grating pattern structure by definition. Second, if the defect's effects are changing with delay, and they were what is dominating the signal instead of our model, then at least the qualitative finding of a reduced replication (case 1) and increased opacity (case 2 ) with increased delay would still remain true, though now the quantitative model would be wrong. However, especially the $\mathrm{Si}-\mathrm{Cu}$ compound targets of case 1 , due to easier and experienced production routines, are almost perfect with virtually no imperfections as visible on the few ten nm level.

We assume imperfect gratings with $\chi=0.7$, with $\mathrm{CuO}$, hydro-carbon-oxide (both thickness $5 \%$ of $\hat{z}_{0}$ ) and $\mathrm{SiO}$ (thickness $0.3 \%$ of $\hat{z}_{0}$ ) surface contamination layers at all applicable surfaces. We further assume $\varepsilon=10 \%$ normal distributed random variations in all the geometric parameters: ridge edge positions, ridge heights, layer thicknesses and smoothnesses, contamination thicknesses, with $n=100$ subvolumina with different parameters on a target in the XFEL focus (modeling and stability analysis for these choices see the Supplemental Material, Fig. 17 [25]).

\section{Optical laser}

The experiments were performed at the Matter in Extreme Conditions (MEC) endstation of the Linac Coherent Light Source (LCLS) at the Stanford Linear Accelerator Center (SLAC). We used the MEC short-pulse HI pump laser to generate a solid-density plasma, i.e., induce fast, nonthermal melting and ionization/excitation. This is a titanium:sapphirebased high power laser system based on chirped pulse amplification. The optical and XFEL configuration for case 1 is the same as described in [17], i.e., the optical pump laser provided ultrashort pulses ( $\tau=80 \mathrm{fs}$ ) at a central wavelength of $800 \mathrm{~nm}$ with an energy of 0.4 or $1 \mathrm{~J}$ before the compressor which corresponds to 180 and $460 \mathrm{~mJ}$ on target, focused to a spot size of $30 \mu \mathrm{m} \times 16 \mu \mathrm{m}$ FWHM.

For case 2 the laser was compressed and focused more tightly ( $\tau=40 \mathrm{fs}$, spot size FWHM $5 \mu \mathrm{m}$ ) resulting in two orders of magnitude higher peak intensity on target of approximately $2-4 \times 10^{19} \mathrm{~W} / \mathrm{cm}^{2}$.

\section{XFEL}

The LCLS XFEL beam was used to diagnose the plasma dynamics by means of SAXS. We used two ranges of $\mathrm{x}$ ray photon energies: off-resonant, i.e., $7990 \mathrm{eV}$ (case 1) and $7940 \mathrm{eV}$ (case 2) and resonant, i.e., $8065 \mathrm{eV}$ (case 1), $8165 \mathrm{eV}$ (case 2). At the off-resonant $\mathrm{x}$-ray energies, the cross sections are dominated by photoionization (bf transitions) and Thomson scattering. Photon energies on $\mathrm{Cu}-K \alpha$ bb resonance transitions allowed us to probe the ionization state of $\mathrm{Cu}$ via $\mathrm{x}$-ray absorption. The fundamental frequency of the LCLS $\mathrm{x}$-ray beam was focused with compound refractive lenses into the MEC experimental area to spot sizes of $20 \mu \mathrm{m}$ (case 1) and between 5 and $10 \mu \mathrm{m}$ (case 2). The third harmonic was only weakly focused and hence its intensity is significantly reduced on target. For case 2 a high harmonic rejection mirror system was used. While in case 1 the XFEL pulse intensity had to be attenuated by various $\mathrm{Si}$ and $\mathrm{Cu}$ absorbers to ensure the scattering signal was within the dynamic range of the PIXIS XF 2048B camera, for case 2 we positioned small absorber plates in front of the camera to selectively attenuate the first scattering peaks only. This allowed us to use much higher XFEL transmissions (between 20\% and 100\%) than reported in [17]. Consequently, we could measure the signal to higher values of $q$. The delay time error of \pm 114 fs between the optical laser and the XFEL is given by the rms jitter of the XFEL.

\section{Small-angle $x$-ray scattering}

We used a PIXIS XF 2048B x-ray camera to record the scattering pattern. For absolute photon numbers we calibrated it using an $\mathrm{Am}^{241}$ and an $\mathrm{Fe}^{55}$ source. The system resolution is dictated by the PIXIS point spread function and XFEL beam divergence, which are both between 2 and 3 pixels on the detector.

\section{5. $K \alpha$ emission spectra}

The presence of nitrogenlike ions and therefore the resonant absorption transitions in the $\mathrm{Cu}$ targets (case 2) and their absence in the case of $\mathrm{Si}-\mathrm{Cu}$ compound targets (case 1) was independently verified by $K \alpha$ emission spectra. Those were measured by a spectrometer employing a $2 \times 4 \mathrm{~cm}$ large HOPG crystal with a mosaicity of $m=0.8^{\circ}$ observing the rear side of the target. This instrument provides a spectral resolution better then $10 \mathrm{eV}$, the data are spatially and temporally integrated. Figure 9 presents the measured spectra for the two different targets highlighting the $K \alpha$ emission line of $\mathrm{N}$-like $\mathrm{Cu}$ at $8165 \mathrm{eV}$. 


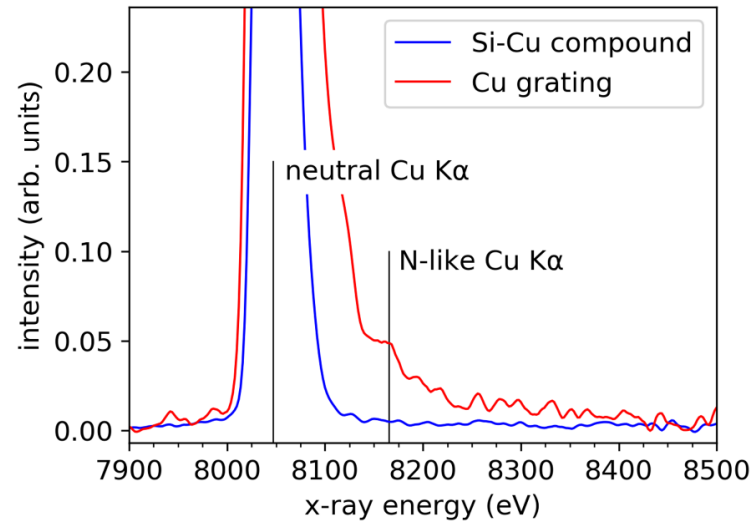

FIG. 9. The measured $K \alpha$ emission spectra for two different target compositions. In the case of a pure $\mathrm{Cu}$ target, the emission at the probe photon energy of $8165 \mathrm{eV}$ is clearly visible showing the presence of Ni-like ions. In contrary, in the $\mathrm{Si}-\mathrm{Cu}$ compound target the ionization of $\mathrm{Cu}$ is by far not so high as most of the laser energy is absorbed in the Si layer.

\section{PIC simulations}

We performed PIC simulations using the collisional PIC code SMILEI [45]. This code treats binary collisions within a cell between charged particles and includes ionization via field ionization and collisional direct impact ionization [46]. It does not include recombination, hence the simulation gets unphysical when the plasma is first heated and then is cooled down too much, which is the case in case 2 after a few hundred fs. The spatial and temporal resolution for the simulations was set to $\Delta x=\Delta y=c \Delta t / \sqrt{2}=\lambda_{\text {laser }} / 400$ for case 1 and $\lambda_{\text {laser }} / 800$ for case 2 . The simulation box was set to $3.6 \mu \mathrm{m}$ in $x$ direction (laser direction) for case $1,1.6 \mu \mathrm{m}$ for case 2 , and $200 \mathrm{~nm}$ in transverse $y$ direction. The laser polarization was set in $y$ direction, and the transverse envelope was a planar. We took the finite waist into account by running the simulation several times with different laser intensities and averaging the respective contribution to the asymmetry. The other laser parameters are set to experimental values shown in Fig. 1(b) in the main text. We placed 20 ions per cell, copper being preionized to charge state $1+$, and added electrons to start with a neutral plasma. All other geometric and laser parameters were set according to Fig. 1 in the main text.

\section{Hydrodynamic simulations}

The hydrodynamic simulations in case 1 were performed in PALE2 code [47]. It is a two-dimensional arbitrary Lagrangian Eulerian code for laser plasma simulations, but only the Lagrangian steps (i.e., the computational mesh following the flow of the matter) were applied, in order to maintain the interface between the materials perfectly resolved. The initial condition was given by the PIC results at time 0.5 ps, which were integrated over the velocity space and averaged in the transversal direction to eliminate the sampling noise of the particle method. The only exception from the rule was the density and material profile and the corresponding mesh shape, where the analytic formulas for the buried and rear interfaces were used. The computational mesh consisted of 100 cells in the transversal and 200 cells in the longitudinal direction, where exactly half of them was dedicated to each of the material parts considering their different densities. The mesh was nonuniform with the spatial step geometrically decreasing towards the interfaces with the coefficient 0.96 , in order to model their thermal motion in detail. In addition to the two-temperature hydrodynamics, the electron heat diffusion model was employed, where the Spitzer-Härm formula (adopted from [48]) was corrected for the dense and low temperature plasma according to [49] based on the bulk solid heat conductivities. The radiation transport was found to have insignificant effects on the dynamics for the given range of temperatures, so it was omitted in the simulations for simplicity and consistency with the PIC modeling.

\section{Atomic rate simulations}

The opacity of the trajectory shown in Fig. 7(f) by the orange arrow was estimated by running simulations using the toolkit SCFLY [31] with different non-LTE temperatures at a collisional radiative steady state. The resulting ionization distributions were compared to that of the PIC simulation at each time step and the SCFLY simulation with the best agreement was chosen. In a second step, those temperatures were used for the opacity calculation using SCFLY-spec in order to obtain an approximate temporal evolution of the expected plasma opacity.

\section{Calculation of the experimental average asymmetry}

The raw data PIXIS signal was summed within a square of 17 pixels width for each individual scatter peak and then corrected by the corresponding mean of two background values determined at both sides of the signal axis. The result was normalized to photon numbers $N_{\text {ph }}$ by a conversion factor of $1 / 164$ photons per ADU which was calibrated off-line (as in [17]). The total uncertainty is given by the Poisson error $\sqrt{N_{\mathrm{ph}}}$, the statistical background error given by the standard deviation $\sigma_{\mathrm{Bg}}$ of 12 background values allocated at both sides of the peaks), and the dark field error $\sigma_{\mathrm{DF}}$ (measured analogously). Applying Gaussian error propagation the individual peak error is then given by $\Delta N_{\mathrm{ph}}=$ $\sqrt{N_{\mathrm{ph}}(q)+\left(\sigma_{\mathrm{Bg}}-\sigma_{\mathrm{DF}}\right)^{2}+\left(\sigma_{\mathrm{DF}}\right)^{2}}$. For each pair of scattering peaks situated at $+q_{i}$ and $-q_{i}$ with photon numbers $N_{\mathrm{ph}, i}^{+}$and $N_{\mathrm{ph}, i}^{-}$, respectively, the individual asymmetry was calculated according to $\eta_{i}=\left(N_{\mathrm{ph}, i}^{+}-N_{\mathrm{ph}, i}^{+}\right) /\left(N_{\mathrm{ph}, i}^{+}+N_{\mathrm{ph}, i}^{-}\right)$. This peakwise asymmetry was then averaged over all $q_{i}$ in the $q$ range that is experimentally accessible, which helps to reduce the error margin in the experiment compared to the single measurement $\eta_{i}$. The error of the average asymmetry is then again derived by applying Gaussian error propagation.

In case 1 we averaged the asymmetry $\left|\eta_{0}\right|$ over the experimentally accessible $q$ range from $q=0.05 \mathrm{~nm}$ to $0.15 / \mathrm{nm}$, preshot asymmetries are around $0.13 \pm 0.5$. The accessible $q$ range in case 2 was larger due to the use of absorbers, $q \approx 0.015 \mathrm{~nm}$ to $0.2 / \mathrm{nm}$. The average preshot asymmetry here ranged from 0.03 to 0.09 with uncertainties in the range from 0.01 to 0.06 . 


\section{Statistical significance}

To determine the statistical significance of the resonant main shots on copper being more asymmetric than the preshots and the off-resonance shots being less asymmetric, we calculated the conditional probability of the respective shots being either all more asymmetric $\left(p_{>}\right)$or all less asymmetric $\left(p_{<}\right)$. Here $p_{>}=\prod_{j \in M} p_{>}^{j}$ is the product of the individual probabilities for each shot $j$ of the set $M$ of res- onant or off-resonant shots. For example, the probability for the asymmetry being increased on the resonant main shots is given by $p_{>} /\left(p_{<}+p_{>}\right)$. To calculate $p_{>}^{j}\left(p_{<}^{j}\right)$ we assume a Gaussian distribution of the probability density distribution for the real value being around the measured value, with the width of the Gaussian given by the respective measurement error bar, and integrate from $\eta / \eta_{0}=1$ to $\infty$ (from 0 to 1 ). The result is $p_{>} /\left(p_{<}+p_{>}\right)=0.999999902$ for the resonant shots with delays equal to or less than $300 \mathrm{fs}$, which is larger than the $5 \sigma$ level.
[1] D. Kraus, J. Vorberger, A. Pak, N. J. Hartley, L. B. Fletcher, S. Frydrych, E. Galtier, E. J. Gamboa, D. O. Gericke, S. H. Glenzer et al., Formation of diamonds in laser-compressed hydrocarbons at planetary interior conditions, Nat. Astron. 1, 606 (2017).

[2] B. A. Remington, Modeling astrophysical phenomena in the laboratory with intense lasers, Science 284, 1488 (1999); S. V. Bulanov, T. Z. Esirkepov, M. Kando, J. Koga, K. Kondo, and G. Korn, On the problems of relativistic laboratory astrophysics and fundamental physics with super powerful lasers, Plasma Phys. Rep. 41, 1 (2015).

[3] S. Le Pape, L. F. Berzak Hopkins, L. Divol, A. Pak, E. L. Dewald, S. Bhandarkar, L. R. Bennedetti, T. Bunn, J. Biener, J. Crippen et al., Fusion Energy Output Greater than the Kinetic Energy of an Imploding Shell at the National Ignition Facility, Phys. Rev. Lett. 120, 245003 (2018).

[4] E. E. McBride, A. Krygier, A. Ehnes, E. Galtier, M. Harmand, Z. Konôpková, H. J. Lee, H.-P. Liermann, B. Nagler, A. Pelka et al., Phase transition lowering in dynamically compressed silicon, Nat. Phys. 15, 89 (2019).

[5] J. E. Bailey, T. Nagayama, G. P. Loisel, G. A. Rochau, C. Blancard, J. Colgan, P. Cosse, G. Faussurier, C. J. Fontes, F. Gilleron et al., A higher-than-predicted measurement of iron opacity at solar interior temperatures, Nature (London) 517, 56 (2015).

[6] P. Morel, J. Daugas, G. Gosselin, V. Méot, and D. Gogny, Nuclear excitation by electronic processes: NEEC and NEET effects, Nucl. Phys. A 746, 608 (2004).

[7] D. L. Bleuel, L. A. Bernstein, C. A. Brand, W. S. Cassata, B. H. Daub, L. S. Dauffy, B. L. Goldblum, J. M. Hall, C. A. Hagmann, L. Berzak Hopkins et al., Method for detection of nuclear-plasma interactions in a ${ }^{134}$ Xe-doped exploding pusher at the National Ignition Facility, Plasma and Fusion Research 11, 3401075 (2016)

[8] L. G. Huang, T. Kluge, and T. E. Cowan, Dynamics of bulk electron heating and ionization in solid density plasmas driven by ultra-short relativistic laser pulses, Phys. Plasmas 23, 063112 (2016); T. Kluge, M. Bussmann, H.-K. Chung, C. Gutt, L. G. Huang, M. Zacharias, U. Schramm, and T. E. Cowan, Nanoscale femtosecond imaging of transient hot solid density plasmas with elemental and charge state sensitivity using resonant coherent diffraction, ibid. 23, 033103 (2016).

[9] R. Betti and O. A. Hurricane, Inertial-confinement fusion with lasers, Nat. Phys. 12, 435 (2016).

[10] Z. Y. Ge, W. Yu, H. B. Zhuo, C. T. Zhou, Y. Y. Ma, X. H. Yang, T. P. Yu, D. B. Zou, S. X. Luan, Y. Yin et al., Resonant absorption and not-so-resonant absorption in short, intense laser irradiated plasma, Phys. Plasmas 20, 073301 (2013).

[11] A. Sgattoni, S. Sinigardi, L. Fedeli, F. Pegoraro, and A. Macchi, Laser-driven Rayleigh-Taylor instability: Plasmonic effects and three-dimensional structures, Phys. Rev. E 91, 013106 (2015).

[12] S. Chawla, M. S. Wei, R. Mishra, K. U. Akli, C. D. Chen, H. S. McLean, A. Morace, P. K. Patel, H. Sawada, Y. Sentoku et al., Effect of Target Material on Fast-Electron Transport and Resistive Collimation, Phys. Rev. Lett. 110, 025001 (2013); S. Göde, C. Rödel, K. Zeil, R. Mishra, M. Gauthier, F.-E. Brack, T. Kluge, M. J. MacDonald, J. Metzkes, L. Obst et al., Relativistic Electron Streaming Instabilities Modulate Proton Beams Accelerated in Laser-Plasma Interactions, ibid. 118, 194801 (2017); P. Leblanc and Y. Sentoku, Scaling of resistive guiding of laser-driven fast-electron currents in solid targets, Phys. Rev. E 89, 023109 (2014).

[13] P. Emma, R. Akre, J. Arthur, R. Bionta, C. Bostedt, J. Bozek, A. Brachmann, P. Bucksbaum, R. Coffee, F.-J. Decker et al., First lasing and operation of an Ångstrom-wavelength free-electron laser, Nat. Photonics 4, 641 (2010).

[14] D. Pile, First light from SACLA, Nat. Photonics 5, 456 (2011).

[15] T. Tschentscher, C. Bressler, J. Grünert, A. Madsen, A. Mancuso, M. Meyer, A. Scherz, H. Sinn, and U. Zastrau, Photon beam transport and scientific instruments at the European XFEL, Appl. Sci. 7, 592 (2017).

[16] T. Gorkhover, S. Schorb, R. Coffee, M. Adolph, L. Foucar, D. Rupp, A. Aquila, J. D. Bozek, S. W. Epp, B. Erk et al., Femtosecond and nanometre visualization of structural dynamics in superheated nanoparticles, Nat. Photonics 10, 93 (2016).

[17] T. Kluge, M. Rödel, J. Metzkes-Ng, A. Pelka, A. L. Garcia, I. Prencipe, M. Rehwald, M. Nakatsutsumi, E. E. McBride, T. Schönherr et al., Observation of Ultrafast Solid-Density Plasma Dynamics Using Femtosecond X-Ray Pulses from a Free-Electron Laser, Phys. Rev. X 8, 031068 (2018).

[18] N. Mukharamova, S. Lazarev, J.-M. Meijer, O. Y. Gorobtsov, A. Singer, M. Chollet, M. Bussmann, D. Dzhigaev, Y. Feng, M. Garten et al., Femtosecond laser produced periodic plasma in a colloidal crystal probed by XFEL radiation, Sci. Rep. 10, 10780 (2020).

[19] A. Schropp, R. Hoppe, V. Meier, J. Patommel, F. Seiboth, Y. Ping, D. G. Hicks, M. A. Beckwith, G. W. Collins, A. Higginbotham et al., Imaging shock waves in diamond with both high temporal and spatial resolution at an XFEL, Sci. Rep. 5, 11089 (2015). 
[20] J. P. Geindre, A. Mysyrowicz, A. D. Santos, P. Audebert, A. Rousse, G. Hamoniaux, A. Antonetti, F. Falliès, and J. C. Gauthier, Frequency-domain interferometer for measuring the phase and amplitude of a femtosecond pulse probing a laserproduced plasma, Opt. Lett. 19, 1997 (1994).

[21] S. Mondal, A. D. Lad, S. Ahmed, V. Narayanan, J. Pasley, P. P. Rajeev, A. P. L. Robinson, and G. R. Kumar, Doppler Spectrometry for Ultrafast Temporal Mapping of Density Dynamics in Laser-Induced Plasmas, Phys. Rev. Lett. 105, 105002 (2010).

[22] M. Bocoum, F. Böhle, A. Vernier, A. Jullien, J. Faure, and R. Lopez-Martens, Spatial-domain interferometer for measuring plasma mirror expansion, Opt. Lett. 40, 3009 (2015).

[23] S. H. Glenzer and R. Redmer, X-ray Thomson scattering in high energy density plasmas, Rev. Mod. Phys. 81, 1625 (2009).

[24] C. Song, R. Bergstrom, D. Ramunno-Johnson, H. Jiang, D. Paterson, M. de Jonge, I. McNulty, J. Lee, K. Wang, and J. Miao, Nanoscale Imaging of Buried Structures with Elemental Specificity Using Resonant X-Ray Diffraction Microscopy, Phys. Rev. Lett. 100, 025504 (2008).

[25] See the Supplementary Material at http://link.aps.org/ supplemental/10.1103/PhysRevResearch.3.043194 for additional information on the experimental data, simulations performed for case 2, and the quantitative analytical modeling of the asymmetry in SAXS patterns and its connection to layer surface replications and ionization for certain cases including those used in the experiment.

[26] L. G. Huang, M. Bussmann, T. Kluge, A. L. Lei, W. Yu, and T. E. Cowan, Ion heating dynamics in solid buried layer targets irradiated by ultra-short intense laser pulses, Phys. Plasmas 20, 093109 (2013).

[27] A. Andreev, N. Kumar, K. Platonov, and A. Pukhov, Efficient generation of fast ions from surface modulated nanostructure targets irradiated by high intensity short-pulse lasers, Phys. Plasmas 18, 103103 (2011).

[28] This assumption is valid for our experiments since as shown in the Supplemental Material [25] the preshots of the same target show similar asymmetry to each other. Therefore, the effect of the phase on the asymmetry, if present at all, can be assumed to be constant, i.e., all changes of the asymmetry between preand main shots cannot be attributed to the influence of a phase, which will therefore for reasons of simplicity be neglected throughout the paper.

[29] M. Šmíd, O. Renner, A. Colaitis, V. T. Tikhonchuk, T. Schlegel, and F. B. Rosmej, Characterization of suprathermal electrons inside a laser accelerated plasma via highly-resolved $K \alpha$ emission, Nat. Commun. 10, 1 (2019).

[30] T. Kluge, C. Rödel, M. Rödel, A. Pelka, E. E. McBride, L. B. Fletcher, M. Harmand, A. Krygier, A. Higginbotham, M. Bussmann et al., Nanometer-scale characterization of laserdriven compression, shocks, and phase transitions, by $\mathrm{x}$-ray scattering using free electron lasers, Phys. Plasmas 24, 102709 (2017).

[31] H. K. Chung, M. H. Chen, and R. W. Lee, Extension of atomic configuration sets of the non-LTE model in the application to the $K \alpha$ diagnostics of hot dense matter, High Energy Density Phys. 3, 57 (2007).

[32] L. G. Huang, H. P. Schlenvoigt, H. Takabe, and T. E. Cowan, Ionization and reflux dependence of magnetic instability generation and probing inside laser-irradiated solid thin foils, Phys. Plasmas 24, 103115 (2017).

[33] S. Kahaly, S. K. Yadav, W. M. Wang, S. Sengupta, Z. M. Sheng, A. Das, P. K. Kaw, and G. R. Kumar, Near-Complete Absorption of Intense, Ultrashort Laser Light by Sub- $\lambda$ Gratings, Phys. Rev. Lett. 101, 145001 (2008).

[34] T. Kluge, C. Gutt, L. G. Huang, J. Metzkes, U. Schramm, M. Bussmann, and T. E. Cowan, Using X-ray free-electron lasers for probing of complex interaction dynamics of ultra-intense lasers with solid matter, Phys. Plasmas 21, 033110 (2014).

[35] J. R. Fienup and A. M. Kowalczyk, Phase retrieval for a complex-valued object by using a low-resolution image, J. Opt. Soc. Am. A 7, 450 (1990).

[36] B. Leshem, R. Xu, Y. Dallal, J. Miao, B. Nadler, D. Oron, N. Dudovich, and O. Raz, Direct single-shot phase retrieval from the diffraction pattern of separated objects, Nat. Commun. 7, 1 (2016).

[37] R. M. P. Tanyag, C. Bernando, C. F. Jones, C. Bacellar, K. R. Ferguson, D. Anielski, R. Boll, S. Carron, J. P. Cryan, L. Englert et al., Communication: X-ray coherent diffractive imaging by immersion in nanodroplets, Struct. Dyn. 2, 1 (2015).

[38] L.-M. Stadler, C. Gutt, T. Autenrieth, O. Leupold, S. Rehbein, Y. Chushkin, and G. Grübel, Hard X Ray Holographic Diffraction Imaging, Phys. Rev. Lett. 100, 245503 (2008).

[39] C. M. Günther, B. Pfau, R. Mitzner, B. Siemer, S. Roling, H. Zacharias, O. Kutz, I. Rudolph, D. Schondelmaier, R. Treusch et al., Sequential femtosecond x-ray imaging, Nat. Photonics $\mathbf{5}$, 99 (2011).

[40] L. Gaus, L. Bischoff, M. Bussmann, E. Cunningham, C. B. Curry, J. E. E. Galtier, M. Gauthier, A. Laso García, M. Garten et al., dataset for this publication, RODARE (2021), doi:10.14278/rodare.597.

[41] www.hibef.eu.

[42] S. Mäder: Oxidation metallischer nanofilme für single-use vakuumsensorik, Ph.D. thesis, Ruhr-Universität Bochum, 2011.

[43] H. Neergaard Waltenburg and J. T. Yates, Surface chemistry of silicon, Chem. Rev. 95, 1589 (1995); C. Hollauer, Modeling of thermal oxidation and stress effects, Ph.D. thesis, Technische Universität Wien, Wien, 2007.

[44] A. Belkind and S. Gershman, Plasma cleaning of surfaces, Vac. Coating Technol., 1 (2008).

[45] J. Derouillat, A. Beck, F. Pérez, T. Vinci, M. Chiaramello, A. Grassi, M. Flé, G. Bouchard, I. Plotnikov, N. Aunai et al., Smilei: A collaborative, open-source, multi-purpose particle-incell code for plasma simulation, Comput. Phys. Commun. 222, 351 (2018).

[46] R. Mishra, P. Leblanc, Y. Sentoku, M. S. Wei, and F. N. Beg, Collisional particle-in-cell modeling for energy transport accompanied by atomic processes in dense plasmas, Phys. Plasmas 20, 072704 (2013).

[47] R. Liska, M. Kuchařík, J. Limpouch, O. Renner, P. Váchal, L. Bednárik, and J. Velechovský, ALE method for simulations of laser-produced plasmas, Springer Proc. Math. 4, 857 (2011).

[48] Y. T. Lee and R. M. More, An electron conductivity model for dense plasmas, Phys. Fluids 27, 1273 (1984).

[49] K. Eidmann, J. Meyer-Ter-Vehn, T. Schlegel, and S. Hüller, Hydrodynamic simulation of subpicosecond laser interaction with solid-density matter, Phys. Rev. E 62, 1202 (2000). 